\title{
Defect-related photoluminescence of hexagonal boron nitride
}

\author{
Luc Museur \\ Laboratoire de Physique des Lasers-LPL, CNRS UMR 7538, Institut Galilée, Université Paris 13, 93430 Villetaneuse, France \\ Eduard Feldbach \\ Institute of Physics, University of Tartu, 142 Riia Street, 51014 Tartu, Estonia \\ Andrei Kanaev \\ Laboratoire d'Ingénierie des Matériaux et des Hautes Pressions-LIMHP, CNRS, Institut Galilée, Université Paris 13,
93430 Villetaneuse, France
}

(Received 19 May 2008; revised manuscript received 2 July 2008; published 16 October 2008)

\begin{abstract}
Photoluminescence of polycrystalline hexagonal boron nitride ( $\mathrm{hBN}$ ) was measured by means of time- and energy-resolved spectroscopy methods. The observed bands are related to donor-acceptor pair transitions, impurities, and structural defects. The excitation of samples by high-energy photons above $5.4 \mathrm{eV}$ enables a phenomenon of photostimulated luminescence (PSL), which is due to distantly trapped conduction band electrons and valence band holes. These trapped charges are metastable and their re-excitation with low-energy photons results in anti-Stokes photoluminescence. The comparison of photoluminescence excitation spectra and PSL excitation spectra allows band analysis that supports the hypothesis of Frenkel-type exciton in hBN with a large binding energy.
\end{abstract}

DOI: 10.1103/PhysRevB.78.155204

PACS number(s): 78.55.Cr, 71.35.-y, 71.55.Eq

\section{INTRODUCTION}

Optical and fluorescence properties of hexagonal boron nitride $(\mathrm{hBN})$ deserved particular interest during the last decade since the first observation of an intense far-UV exciton emission, ${ }^{1,2}$ making this material a candidate for use in new light-emitting devices. A successful synthesis of high-purity single-crystal samples initially at high pressure and temperature $^{1,2}$ and later at atmospheric conditions ${ }^{2}$ enabled this achievement. Accordingly, understanding of the electronic band and exciton structure of hBN becomes even more an important issue as it serves a basic system for single-wall ${ }^{3,4}$ and multiwall boron nitride nanotubes. ${ }^{5-9}$

Despite of many efforts in the past, the electronic properties of hBN remain largely unknown. They have been earlier studied by luminescence, ${ }^{1,10-17}$ optical reflectance and absorption, ${ }^{18-21}$ photoconductivity, ${ }^{22} \mathrm{x}$-ray emission, ${ }^{23-25}$ inelastic x-ray scattering, ${ }^{26-28} \mathrm{x}$-ray absorption, ${ }^{25,29,30}$ and electron energy loss ${ }^{31-33}$ spectroscopy. After all, a large spread of band-gap energies reported in literature, ranging from 3.1 to $7.1 \mathrm{eV},{ }^{14}$ is currently explained by sample quality and related to experimental methods used. Recently, arguing on generally high luminosity of the free exciton luminescence, Watanabe et al. ${ }^{1}$ assumed that $\mathrm{hBN}$ is a direct band-gap material. They have measured the band-gap energy of $5.971 \mathrm{eV}$ and inferred an exciton binding energy of $0.149 \mathrm{eV}$ that corresponds to the Wannier exciton model. However this result is in a large disagreement with the most recent theoretical calculations using the so-called all-electron $G W$ approximation. ${ }^{34,35}$ They predict hBN to be an indirect bandgap material with a band-gap energy of $5.95 \mathrm{eV}$ and a lowest direct interband transitions at $6.47 \mathrm{eV}^{34}$ Moreover, Arnaud et $a l .{ }^{34}$ have deduced a huge exciton binding energy of $0.72 \mathrm{eV}$ and predicted that the low-lying exciton in $\mathrm{hBN}$ belongs to the Frenkel type. The intense free exciton luminescence observed in single-crystal hBN is explained by a large oscilla- tor strength of merged excitonic transitions. ${ }^{34}$

In view of many disagreements further confrontation between experiment and theory will continue. In these conditions, more experimental data concerning electronic and related optical properties of $\mathrm{hBN}$ are highly required. In particular, energy- and time-resolved photoluminescence methods provide valuable information about excitonic and interband transitions. Moreover, in polycrystalline samples energy transfer to impurities and defects may inhibit these intrinsic transitions and strongly affect the fluorescence spectra. However, the energy transfer is specific to excitation energy and the relevant excited states can be experimentally resolved.

In the present paper we report on detailed analysis of the defect-related intraband luminescence of hBN. Important information about intrinsic properties of $\mathrm{hBN}$ is obtained by combining two experimental approaches: time- and energyresolved photoluminescence and photostimulated luminescence. The discussion is organized as follow. The experiment is described in Sec. II and experimental results are presented on the Sec. III. In Sec. IV A we discuss the nature of the observed luminescence bands. Finally, in Sec. IV B, careful comparison between photoluminescence excitation (PLE) and photostimulated luminescence (PSL) excitation spectra brings more precision to exciton and band-gap transitions of hexagonal BN.

\section{EXPERIMENT}

The samples were prepared from commercial hexagonal BN powders (Alfa 99.5\%) compacted in pellets of size 8 $\times 8 \times 1 \mathrm{~mm}^{3}$ at a hydrostatic pressure of $0.6 \mathrm{GPa}$. The grit size of the hBN powder has been estimated by means of granulometry and transmission electron microscopy (JEM $100 \mathrm{C}$ JEOL). It ranged from 0.3 to $10 \mu \mathrm{m}$ with an average 
particle size of $3.1 \mu \mathrm{m}$ corresponding to the maximum in the mass distribution curve. The samples were then heated at $800 \mathrm{~K}$ under vacuum for $12 \mathrm{~h}$ to avoid organic impurities and traces of water.

The luminescence properties of the samples were studied using vacuum ultraviolet (VUV) synchrotron-radiation (SR) source of DORIS storage ring at HASYLAB (DESY, Hamburg). The facility of the SUPERLUMI station used in the experiments is described in details elsewhere. ${ }^{36}$ Briefly, samples were cooled down to $8 \mathrm{~K}$ and irradiated by monochromatized SR $(\Delta \lambda=3.3 \AA)$ under high vacuum $\left(\sim 10^{-9}\right.$ mbar $)$. The measurements of luminescence spectra were carried out using a visible 0.275 -m triple-grating ARC monochromator equipped with a CCD detector or a photomultiplier operating in the photon-counting mode. The pulse structure of SR (130 ps and $5 \mathrm{MHz}$ repetition rate) enables time-resolved luminescence analysis at time scale of $200 \mathrm{~ns}$ with subnanosecond temporal resolution. Spectra were recorded within a time gate $\Delta \tau$ delayed after the SR excitation pulse. Typically two time gates have been used simultaneously: a fast one with $\Delta \tau_{1}=1-4$ ns and a slow one with $\Delta \tau_{3}=22-200 \mathrm{~ns}$. Complementary, luminescence decay curves were measured at fixed excitation and luminescence photon energies. The recorded spectra were corrected for the primary monochromator reflectivity and SR current.

The PSL excitation spectra of $p d$-hBN samples were measured at the BL 52 beamline of MAX-laboratory synchrotron (Lund, Sweden). The experiment built up by the Tartu group is described in Refs. 37 and 38. At each excitation energy $E_{\text {exc }}$ the sample was irradiated by a fixed number of UV or VUV photons $\left(2.5 \times 10^{6}\right.$ counts $)$ in the energy region of $E_{\text {exc }}=5-15 \mathrm{eV}$. After completing the dose, the irradiation was stopped and the phosphorescence decay was measured. When the phosphorescence intensity drops until almost zero [photomultiplier (PM) noise level] that typically happens after a few minutes, the sample was activated by a bright spectroscopic source at $h \nu=2.0 \pm 0.5 \mathrm{eV}$ through a double-prism monochromator DMR-4 and the time dependence of PSL intensity was recorded. The integral PSL intensity was taken as a measure of the number of recombined electron-hole pairs.

\section{RESULTS}

Under photoexcitation above $6 \mathrm{eV}$ the luminescence spectra of $\mathrm{hBN}$ are composed of several luminescence bands (Fig. 1). Basically, one broadband and one structured emissions between 3 and $4 \mathrm{eV}$ and two relatively narrow near band-gap emissions at 5.3 and $5.5 \mathrm{eV}$ were distinguished. Two high-energy emissions were discussed in details in our recent publication. ${ }^{17}$ They were assigned, respectively, to quasi-donor-acceptor pairs (DAPs) ( $q$-DAPs) (5.3 eV band) and bound excitons ( $5.5 \mathrm{eV}$ band). Below we complete the assignment of low-energy luminescence.

\section{A. Luminescence around $4 \mathrm{eV}$ in $\mathrm{hBN}$ powder samples}

The hBN powder sample is a strongly luminescent material, whose luminescence spectra depend on excitation en-

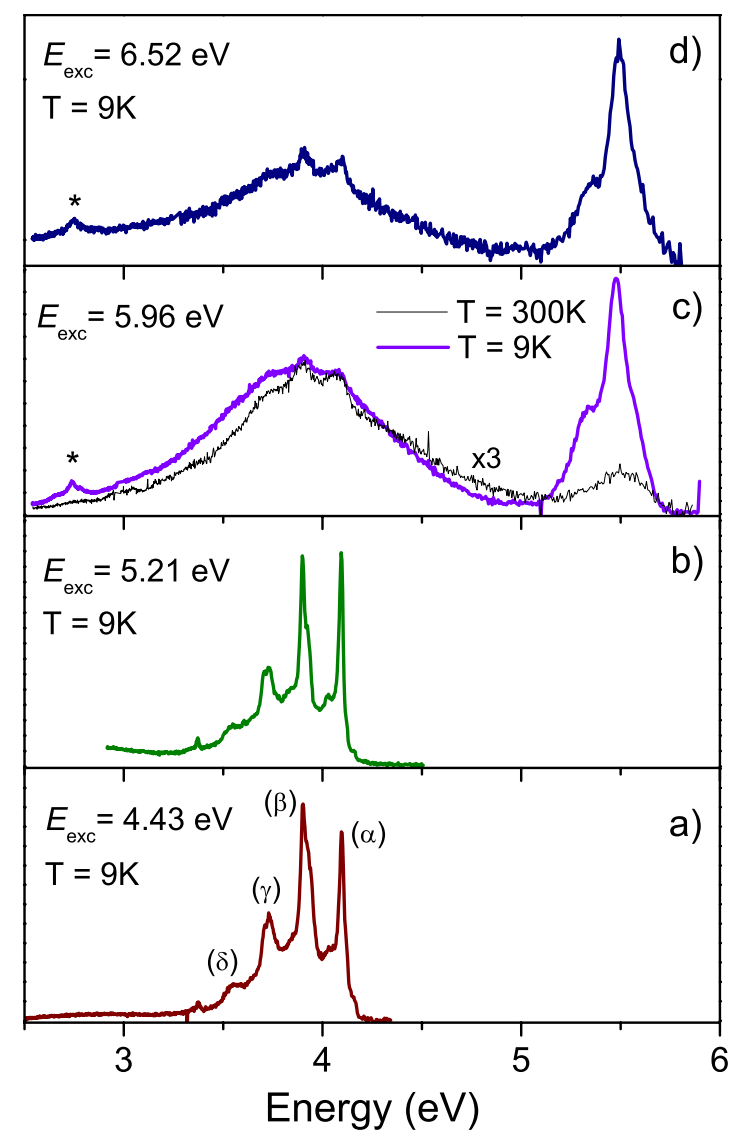

FIG. 1. (Color online) Luminescence spectra of polycrystalline $\mathrm{hBN}$. The excitation energy is indicated on each spectrum. The phonons replicas are labeled in (a). The star in (c) and (d) indicates the second order of the $5.5 \mathrm{eV}$ band.

ergy. The low-temperature luminescence spectra of hBN samples recorded with different excitation energies varying from 4 to $6.5 \mathrm{eV}$ are presented in Fig. 1. Under excitation below $5.0 \mathrm{eV}$ a strong structured UV emission is observed. Four peaks, labeled $(\alpha),(\beta),(\gamma)$, and $(\delta)$, are clearly visible. A multiple Gaussian fit procedure results in the peak energies of $4.099(\alpha), 3.912(\beta), 3.731(\gamma)$, and $3.539 \mathrm{eV}(\delta)$. These four peaks are equally spaced in energy by $w_{g}$ $=186 \pm 1.4 \mathrm{meV}$. At room temperature these peaks are broadened but no appreciable shift was detected. When the excitation energy exceeds $5.0 \mathrm{eV}$, a very broad band $(\Delta E$ $\sim 1 \mathrm{eV}$ ) centered at $3.9 \mathrm{eV}$ appears and superimposes with the structured emission [Figs. 1(c) and 1(d)]. However, the peaks of the structured emission can always be observed on the top of the broad band.

At excitation energy of $4.27 \mathrm{eV}$ only the structured UV emission can be observed. The luminescence decay curves of its four peaks $(\alpha)-(\delta)$ are shown in Fig. 2. Within the experimental error bars, all the decay curves are single exponential with short characteristic decay time of $\tau=1.1$ ns. The typical decay curve of the broad emission measured at $E_{\text {lum }}$ $=3.91 \mathrm{eV}$ and $E_{\mathrm{exc}}=5.96 \mathrm{eV}$ is plotted in Fig. 2 by curve $(\varepsilon)$. At this excitation energy the broad band dominates the luminescence spectra. Although the probed energy $E_{\text {lum }}$ $=3.91 \mathrm{eV}$ is in coincidence with the peak $(\beta)$ of the structured emission, the decay curve appears to be multiexponen- 


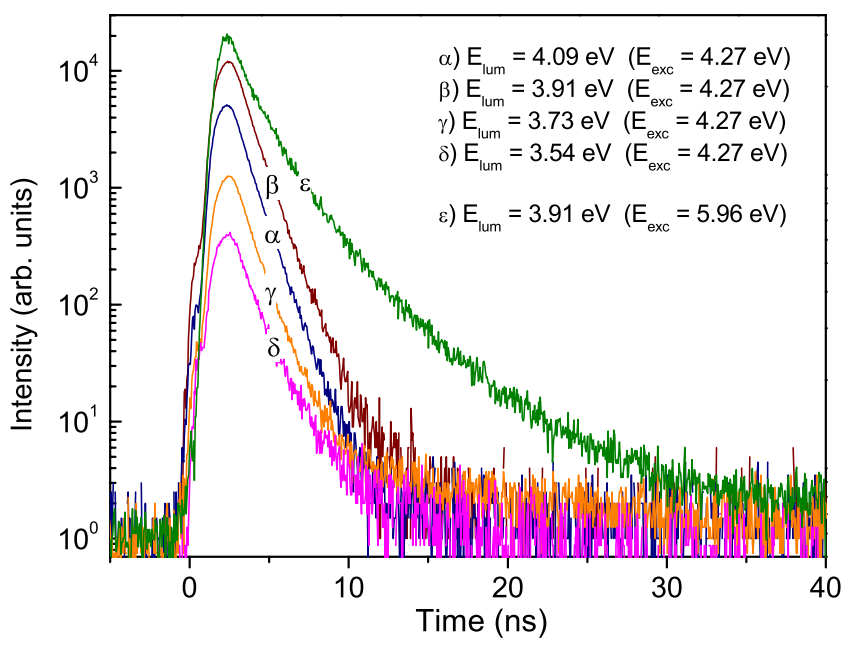

FIG. 2. (Color online) Luminescence decay curves at different photon energies. Curves $\alpha, \beta, \gamma$, and $\delta$ correspond to the peaks of the structured emission. Curve $\varepsilon$ corresponds to the broad emission maximum.

tial and of longer lifetime than that of the peak $(\beta)$.

The time-resolved photoluminescence method allows separating several contributions of superposed luminescence bands if their respective lifetimes are noticeably different. This is the case of the structured and continuous broadband emissions around $4 \mathrm{eV}$. Figure 3(a) shows the total PLE spectrum measured at $E_{\text {lum }}=3.73 \mathrm{eV}$. The last corresponds to the peak $(\gamma)$ of the structured emission and is close to the maximum of the broadband emission. Two principal features can be distinguished in this spectrum: (i) a sharp onset at $4.09 \mathrm{eV}$ followed by a decrease in the luminescence intensity until nearly zero at $E_{\mathrm{exc}}=4.9 \mathrm{eV}$ and (ii) a strong peak at $5.95 \mathrm{eV}$ preceded by a weak structured footer that begins at $5.0 \mathrm{eV}$. The PLE spectra obtained with short- and long-time windows are displayed in Figs. 3(b) and 3(c). The long-time window of $\Delta \tau_{2}=22-200 \mathrm{~ns}$ selects long-lived excited states, which are apparently responsible for the broad emission band [Fig. 3(c)]. The peak maximum at $5.95 \mathrm{eV}$ strongly contributes to excitation of this band. This peak is blueshifted with respect to that of the exciton absorption at 5.82 $\mathrm{eV}$ in $\mathrm{hBN}$ single crystal. ${ }^{1}$ Nevertheless, a weak luminescence at $5.9 \mathrm{eV}$ is also reported in $\mathrm{hBN}$ single crystal at low temperatures and assigned to another exciton series. The Stokes shift with respect to the excitation peak at $5.95 \mathrm{eV}$ could result from inhomogeneities of the local field or dislocations such as stacking faults. ${ }^{1}$ Therefore we tentatively assign the peak at $5.95 \mathrm{eV}$ to the excitation of higher exciton series. This excitonic peak is preceded by a weak and unstructured footer ranging from 5 to $5.5 \mathrm{eV}$ and is followed by a broad continuum until 7.6 eV. Conversely, the PLE spectra obtained with the short-time window $\left(\Delta \tau_{1}=1-4 \mathrm{~ns}\right)$ show contributions from both short- and long-decay luminescence components [Fig. 3(b)]. Nevertheless, a comparison with the PLE spectra of the long component [Fig. 3(c)] allows firmly assigning the structured emission with the onset at $4.09 \mathrm{eV}$ to a short-lived excited state. Moreover, we note a second weak excitation onset of the structured emission at $E_{\text {exc }}=5.2 \mathrm{eV}$.

Similar luminescence spectra to those depicted in Fig. 1 have been recently reported on photoluminescence and

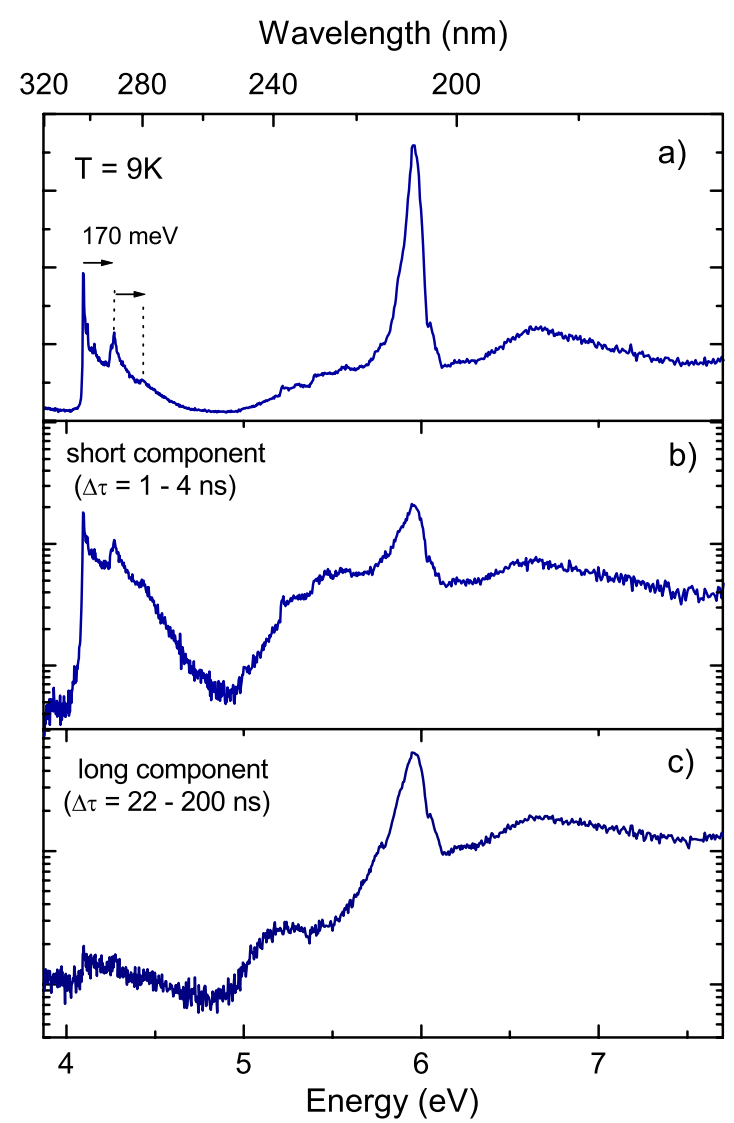

FIG. 3. (Color online) (a) PLE spectra $\left(E_{\text {lum }}=3.72 \mathrm{eV}\right)$ of polycrystalline hBN and its (b) short- and (c) long-lived components.

cathodoluminescence experiments of commercial hBN powder. ${ }^{10,39-48}$ However, in these publications, no distinctions were made between the broad band and structured emissions. We now assign the structured UV emission to impurities (probably C) and the broadband emission to deep DAP of strongly localized center. These assignments will be discussed below in Sec. IV A.

\section{B. Photostimulated luminescence in $\mathrm{hBN}$ powder samples}

The photostimulated luminescence arises from the trapping of free charge carriers in distant lattice sites with subsequent recombination of carriers released from these traps by a visible light. ${ }^{38}$ The charge separation and trapping can result from different excitation processes listed below and depicted in Fig. 4:

$$
\begin{gathered}
A^{-}+D^{+}+h v_{\mathrm{exc}} \rightarrow A^{-}+D^{+}+e^{-}+h^{+} \rightarrow A^{o}+D^{o}, \\
A^{-}+D^{+}+h v_{\mathrm{exc}} \rightarrow A^{o}+D^{+}+e^{-} \rightarrow A^{o}+D^{o}, \\
A^{-}+D^{+}+h v_{\mathrm{exc}} \rightarrow A^{-}+D^{o}+h^{+} \rightarrow A^{o}+D^{o}, \\
A^{-}+D^{+}+h v_{\mathrm{exc}} \rightarrow A^{o}+D^{o} .
\end{gathered}
$$

The band-to-band transitions [Eq. (1)] or impurity-band ionization [Eqs. (2) and (3)] are the most likely contributions to PSL. In these cases at least one of photoexcited charge car- 


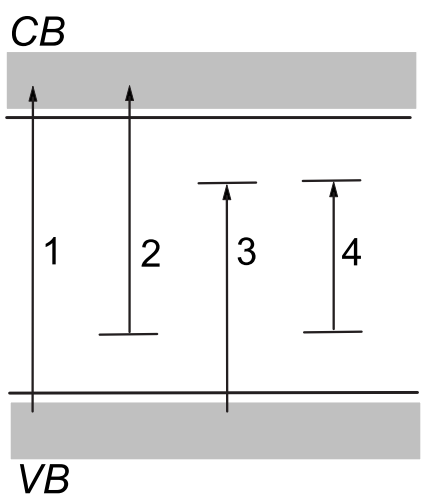

FIG. 4. Schematic representation of electronic transitions relevant to PSL.

riers $\left(e^{-}\right.$or $\left.h^{+}\right)$becomes free and can migrate away from the point of excitation before being trapped by the acceptor or donor defects. Spatially closely trapped charges then annihilate giving rise to phosphorescence. In contrast, at rather large distance their lifetime becomes infinite and their recombination can only be possible after reactivation by light or thermally. Figure 5 shows the phosphorescence decay curves $\left(E_{\text {lum }}=3.91 \mathrm{eV}\right)$ for different photon excitation energies ranging from 5.4 to $5.9 \mathrm{eV}$. At $E_{\text {exc }} \leq 5.50 \mathrm{eV}$ the phosphorescence decay curves are characterized by an almost monoexponential decay with characteristic time of $\tau=5.8 \mathrm{~s}$ (the dotted line in Fig. 5 shows the PM dark noise). However, at $E_{\text {exc }} \geq 5.60 \mathrm{eV}$ another extremely long-lived component of low intensity appears, which looks like a plateau in Fig. 5. We ascribe this long component to the recombination of charges trapped at large distance tending to infinity.

The observed phosphorescence might also be related to the "dark" (dipole forbidden) exciton states theoretically predicted in hBN by Wirtz et al. ${ }^{35}$ The authors suggested a coupling between the "white" (dipole allowed) and dark exciton states, which makes them appear in the absorption spectra of hBN monocrystals reported by Watanabe et al. ${ }^{1}$ However, we disregard their contribution in PSL experiments since the phosphorescence is already observed at $E_{\text {exc }}$ $=5.1 \mathrm{eV}$ (Fig. 5), which is well below the exciton energy in

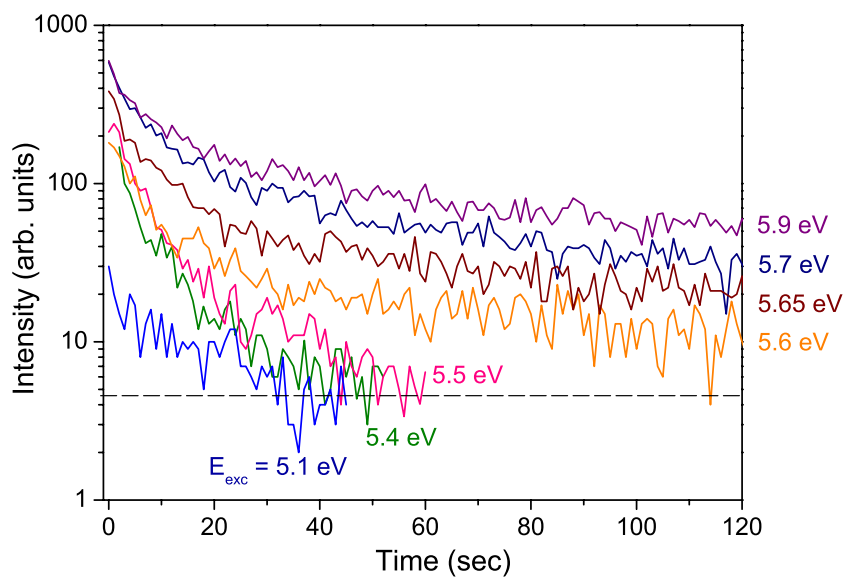

FIG. 5. (Color online) Phosphorescence decay curves for different photon excitation energies.

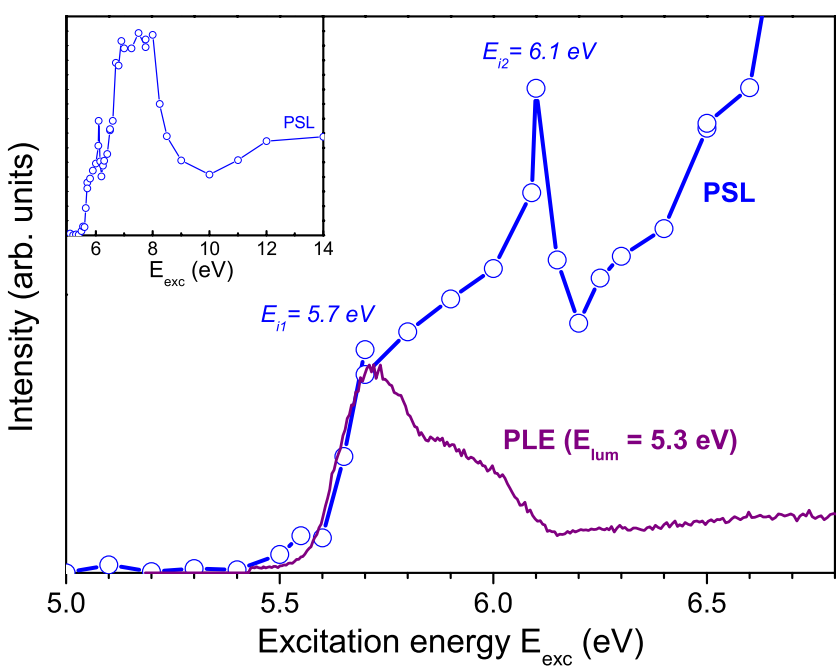

FIG. 6. (Color online) PSL excitation spectrum (open circles) and PLE spectrum of $q$-DAP luminescence $(5.3 \mathrm{eV})$ of polycrystalline $\mathrm{hBN}$. The total measured PSL excitation spectrum is shown in the inset.

$\mathrm{hBN}$. Moreover, PSL is related to the states with infinite lifetime (excited above $5.5 \mathrm{eV}$ ) whose depopulation is only triggered by light. In contrast, the hypothetic dark states become accessible when the crystalline symmetry is broken through defects or limited sample quality. This is our case since no free exciton emission has been observed. Accordingly, the singlet and triplet excitons are expected to be strongly coupled in our polycrystalline $\mathrm{hBN}$ samples and their lifetime is short (subnanosecond). In this case no longlived exciton states are expected.

The PSL excitation spectrum is represented by open circles in Fig. 6. The full measured spectrum is shown in the insert of the figure for indicative purposes. In the following, we will discuss the PSL phenomenon specific to excitation energies ranging from 5 to $7 \mathrm{eV}$. The signal shows up appreciably at $E_{\text {exc }}>5.50 \mathrm{eV}$ in correlation with the appearance of a long component of the phosphorescence decay (Fig. 5). The PSL grows almost exponentially with the excitation energy until $E_{\mathrm{exc}} \equiv E_{i 1}=5.7 \mathrm{eV}$ where it locally attains a maximum. At this energy the slope suddenly changes and the growth of the signal becomes much slower. Another local maximum of the PSL spectrum appears at $E_{\text {exc }} \equiv E_{i 2}$ $=6.10 \mathrm{eV}$. Finally, for excitation energies above $6.2 \mathrm{eV}$ the PSL signal increases progressively until $7 \mathrm{eV}$ where a plateau is reached.

For the sake of the discussion we have superimposed in Fig. 6 the PLE spectrum of the near band-edge luminescence at $E_{\text {lum }}=5.3 \mathrm{eV}$. This emission was discussed in details in our previous publication ${ }^{17}$ and has been assigned to the radiative recombination of the so-called $q$-DAP.

\section{DISCUSSION}

\section{A. Luminescence of polycrystalline hBN samples}

The structured UV luminescence [Figs. 1(a) and 1(b)] has been reported in the past by numerous groups either in 
cathodoluminescence ${ }^{10,43,46,47}$ or photoluminescence experiments. ${ }^{10,14,39-42,44,45,47,48}$ Some authors have assigned it to intrinsic luminescence of $\mathrm{hBN}$ molecular layers ${ }^{47}$ or to phonon-assisted band-edge luminescence. ${ }^{42}$ However, recent experiments ${ }^{1}$ and theoretical calculations ${ }^{34}$ indicate the hBN band-gap energy in the range from 6 to $6.5 \mathrm{eV}$ that disables these interpretations. Others authors $45,49,50$ claimed that the structured luminescence is due to transition between the conduction band (CB) and acceptor carbon atom at substitutional $N$ site. Recently, experimental evidence of the correlation between carbon and oxygen contents in hBN samples and intensity of the structured luminescence has been given. 51

In a recent publication ${ }^{39}$ the structured luminescence of hBN has been attributed to defects or impurities. The peaks $(\beta),(\gamma)$, and $(\delta)$ in Fig. 1(a) were ascribed to phonon replicas of the zero-phonon line $(\alpha)$ involving TO phonons $\left(w_{\mathrm{TO}}=169 \mathrm{meV}\right)$. Due to the low sample temperature in present experiments, the luminescence peaks become well resolved, allowing more precise determination of their spectral positions than in room-temperature experiments. ${ }^{39,42}$ The obtained phonon energy of $w_{g}=186 \mathrm{meV}$, which falls between the known energy of LO $(199 \mathrm{meV})$ and degenerated TO and LO (169 meV) phonons at the $\Gamma$ point, ${ }^{18,19,26,52}$ corresponds more probably to a local phonon mode around the impurity involved in the luminescence process. Moreover, the pronounced red shoulder observed on each phonon replica peak shows that multiphonon processes play a major role in the energy relaxation of the impurity. The coupling between the defect and the lattice is weak as it is shown by the Huang-Rhys factor $S=1.3$ obtained from the normalized peak intensities. This assignment is consistent with fast luminescence decay of the peaks $(\alpha),(\beta),(\gamma)$, and ( $\delta)$ (Fig. 2).

The PLE spectrum shown in Fig. 3(b) supports our interpretation of the defect-related luminescence. Indeed, it reflects characteristic features of such transitions. The luminescence intensity steeply increases and then decreases progressively with the excitation energy. The sharp onset in the PLE spectrum at $4.09 \mathrm{eV}$ corresponds to the minimum energy required to excite the impurity. As in the case of weak electron-phonon coupling, this absorption onset coincides with the zero-phonon luminescence peak $(\alpha)$. Interestingly, two phonon replicas separated by $w_{e}=170 \mathrm{meV}$ that is close to the energy of the TO- and LO-degenerated phonons ${ }^{26}$ can be observed in the PLE spectra. They are indicated by arrows in Fig. 3(a). We see that the energies of phonons involved in the luminescence $\left(w_{g}\right)$ and excitation $\left(w_{e}\right)$ processes are different. This results from the electronic state of the defect, which is not the same in both cases. Consequently, lattice deformations around the defect are different, which affects the local phonon frequencies. The fact that $\omega_{g}>\omega_{e}$ signifies a stronger matrix interaction with the impurity in the excited state.

We do not evidence the impurity involved in the luminescence process in current experiments. However, we can guess about the influence of carbon with more or less confidence. Recent experiments reveal that the structured luminescence strongly shows up in hBN samples contaminated by carbon and oxygen. ${ }^{51}$ This result is consistent with our previous results ${ }^{39}$ and is supported by our complementary photoluminescence experiments carried out with a pyrolytic $\mathrm{BN}$ (pBN) sample. These experiments will be described in details in a forthcoming article. ${ }^{53}$ pBN is a high-purity material, free of carbon compound, obtained by gas-phase reactions between $\mathrm{BCl}_{3}$ and $\mathrm{NH}_{3}$ at $2300 \mathrm{~K}$ and deposition on a Si substrate [chemical vapor deposition (CVD) method]. Actually, pBN sample exhibits no structured emission around 4 $\mathrm{eV}$ whatever the excitation energy is. Its excitation by energy photon above $4.5 \mathrm{eV}$ uniquely results in a continuous emission band similar to that observed in $\mathrm{hBN} .{ }^{53}$ Moreover, the PLE spectrum of hBN (Fig. 3) is rather complicated with a first edge at $4.09 \mathrm{eV}$ and a second one at $5.2 \mathrm{eV}$. This may indicate that the defect involved in the radiative process has at least two excited levels separated by $1.1 \mathrm{eV}$ positioned within the band gap. Since the substitutional carbon impurity at nitrogen site $\left(\mathrm{C}_{N}\right)$ is supposed to introduce two energy levels split by $\sim 0.8 \mathrm{eV}$ in the energy range of $3.2-4.9 \mathrm{eV}$ below the conduction band of $\mathrm{hBN},{ }^{50}$ its participation in the luminescence process is most likely.

We discuss now the nature of the broad luminescence band observed in $\mathrm{hBN}$ powder sample under photoexcitation above $5 \mathrm{eV}$. Due to its long and multiexponential decay [curve $(\varepsilon)$ in Fig. 2], we assign this luminescence to radiative recombination of deep DAPs:

$$
A^{o}+D^{o} \rightarrow A^{-}+D^{+}+h v_{\mathrm{DAP}}
$$

We remark that this deep DAP is different from that related to the 5.3-eV emission ( $q$-DAP). ${ }^{17}$ As Fig. 3(c) shows, the correspondent PLE spectrum of the long-lived luminescence component is dominated by the excitonic peak at $5.95 \mathrm{eV}$. This fact indicates that the energy transfer to the DAP recombination channel via free excitons is efficient,

$$
\begin{gathered}
\mathrm{hBN}+h v_{\mathrm{exc}} \rightarrow \text { exciton, } \\
\text { exciton }+A^{-}+D^{+} \rightarrow A^{0}+D^{0} .
\end{gathered}
$$

This excitonic peak is preceded by a weak shoulder for excitation energies ranging from 5 to $5.5 \mathrm{eV}$. We have assigned this shoulder to a direct excitation of the deep DAP [Eq. (4)]. The PSL spectrum displayed in Fig. 6 shows that no noticeable photostimulated luminescence can be observed following $\mathrm{hBN}$ irradiation between 5 and $5.5 \mathrm{eV}$ that indicates no efficient population of distant traps. In contrast to the direct ionization of the donor or acceptor [Eqs. (2) and (3)], mechanism (4) does not lead to the charge injection into $\mathrm{CB}\left(e^{-}\right)$or valence band $\left(h^{+}\right)$and does not contribute significantly to PSL.

A very large bandwidth of the deep DAP band $(\sim 1 \mathrm{eV})$ and its nearly symmetrical shape suggest that at least one of defects involved in the emission is strongly coupled to the lattice. ${ }^{54}$ This assumption is supported by a comparison of the luminescence spectra obtained at room and low $(9 \mathrm{~K})$ temperatures. When the temperature is increased from 9 to $300 \mathrm{~K}$ [Fig. 1(c)] thermally activated quenching is intensified. The intensity of the broad DAP band then drops and the band shifts to the blue. Blueshifts induced by the rises of the temperature have been reported for deep, the so-called "selfactivated," DAP luminescent bands of several semiconductors: $\mathrm{ZnS},{ }^{55,56} \mathrm{GaAs},{ }^{57}$ and $\mathrm{GaN} .{ }^{58}$ The blueshift is generally 
observed when localized complex centers with strong electron-phonon coupling are involved in the luminescence process. The configurational coordinate (CC) model, which takes into account the interaction of such localized center with matrix, predicts a linear shift of the band position with temperature. ${ }^{56}$ The relevant blueshift that results from thermal occupation of vibrational levels associated with the excited state and thermal quenching is due to radiationless recombination of $e^{-}-h^{+}$pairs.

The acceptor complex center involved in such emissions is usually formed by an acceptorlike vacancy adjacent to a donor impurity atom, ${ }^{55-58}$ and it is often called " $A$ center." The emission results from the electron transition from a relatively distant donor to the donor-vacancy acceptor complex. ${ }^{59}$ According to theoretical calculations most stables defects in $\mathrm{hBN}$ are supposed to be boron vacancy $V_{\mathrm{B}}$ for $n$-type conditions and nitrogen vacancy $V_{\mathrm{N}}$ for $p$-type conditions. To the best of our knowledge there are no calculations concerning vacancy-impurity complexes in hBN. Nevertheless, results obtained in cubic $\mathrm{BN}(\mathrm{cBN})$ show that $V_{\mathrm{B}}-C_{\mathrm{B}}$ and $V_{\mathrm{B}}-O_{\mathrm{N}}$ complexes form deep acceptors. Such center in association with the shallow $C_{\mathrm{B}}$ or $O_{\mathrm{N}}$ donor may be responsible for DAP transitions at $3.9 \mathrm{eV}$ observed in $\mathrm{hBN}$ polycrystalline samples.

\section{B. Photostimulated luminescence and band-gap transitions of hBN}

As we have already mentioned, the PSL excitation onset cannot be a measure of band-gap energy. However, in combination with PLE spectra, PSL excitation spectra can provide valuable information concerning exciton and band-edge energy positions. Below we will discuss the PSL excitation in the framework of relevant processes depicted in Fig. 4. Several interesting details can be remarked from the comparison of PLE and PSL excitation spectra displayed in Fig. 6.

Several free exciton transitions in $\mathrm{hBN}$ merge in the dominant peak of the PLE spectrum [Fig. 3(c)] in the range of photon energies between 5.8 and $6.0 \mathrm{eV}^{34}$ One remarkable feature in Fig. 6 is the noncontribution of these excitons to the PSL excitation. In fact, dissociation of excitons is required for a storage of distant charges, which results in PSL. This dissociation is only possible if the gain in energy due to the charge localization is greater than the exciton binding energy. Using this reasoning, we have previously set up the lower limit to the exciton binding energy $D_{e}>0.4 \mathrm{eV} .{ }^{17}$ Two factors additionally contribute to the inhibition of exciton dissociation: its short lifetime and limited spatial extent. We have not observed free exciton luminescence in our hBN polycrystalline sample. The free excitons are rapidly bound to defects where a part of them recombine radiatively. The lifetime of free excitons in $\mathrm{hBN}$ is then small and that reduces their dissociation probability. In a similar way, small exciton radius decreases the dissociation probability. The noncontribution of the free exciton of $\mathrm{hBN}$ to the PSL processes may be an indication of its small radius and tightbinding energy.

The PSL excitation spectrum in Fig. 6 follows the exponential growth of the PLE spectrum of $q$-DAP luminescence at $5.3 \mathrm{eV}$ below $5.7 \mathrm{eV} .{ }^{17}$ The $q$-DAP states apparently contribute to the PSL signal that complements the general scheme in Fig. 4 by the type IV transition corresponding to the direct $q$-DAP excitation [Eq. (4)]. This fact seems surprising since no free charge carriers are created in such process required for a distant charge trapping. Indeed it can be understood if we consider the $q$-DAP transition energy as a function of the pair distance. Closer charge pairs possess a higher transition energy and distant pairs lower. The charge diffusion within the manifold of $q$-DAP levels below ionization can therefore take place toward more distant and hence long-lived states, which contribute to PSL. The existence of the exchange between $q$-DAP traps excited according to Eq. (4) was suggested in our previous paper. ${ }^{17}$

In the same publication we have reported a change in the $q$-DAP population mechanism at $5.7 \mathrm{eV}$. At this energy the $q$-DAP ionization takes place following Eqs. (2) and (3) and results in the regime crossover from Raman to photoluminescence. This can be an ionization of either acceptor or donor states, whichever is higher lying. Higher-energy photons between 5.7 and $6.0 \mathrm{eV}$ efficiently produce hBN excitons; however, they do not appreciably contribute to PSL. This is seen in Fig. 6 in both PLE $\left(E_{\text {lum }}=5.3 \mathrm{eV}\right)$ and PSL spectra. However, another sharp PSL excitation maximum is observed in the high-energy wing of the exciton absorption band at 6.1 $\mathrm{eV}$. We can relate a high efficiency of the distant charge traps population at this energy to the ionization of acceptor or donor states, whichever is lower lying. Resuming, we attribute two spectral features observed at $E_{i 1}=5.7 \mathrm{eV}$ and $E_{i 2}=6.1 \mathrm{eV}$ in the PSL excitation spectrum to the direct ionization of donor and acceptor levels (or vice versa) involved in the $q$-DAP luminescence [processes II and III of Fig. 4 and Eqs. (2) and (3)]:

$$
\begin{aligned}
& E_{\mathrm{A}^{-}}=E_{g}-E_{i 1 \text { or } i 2}, \\
& E_{\mathrm{D}^{+}}=E_{g}-E_{i 2} \text { or } i 1 .
\end{aligned}
$$

Keeping in mind this assignment, we can estimate the bandgap energy of $\mathrm{hBN}$. Indeed, the energy of the $q$-DAP luminescence $E_{q \text {-DAP }}=5.3 \mathrm{eV}$ is given by the relation

$$
E_{q-\mathrm{DAP}}=E_{g}-E_{\mathrm{A}^{-}}-E_{\mathrm{D}^{+}} .
$$

Combining Eqs. (7) and (8) we then obtain

$$
E_{g}=E_{i 2}+E_{i 1}-E_{q-\mathrm{DAP}} \approx 6.5 \mathrm{eV} .
$$

Alternatively, we can assign the spectral feature observed at $E_{i 2}=6.1 \mathrm{eV}$ to the dissociation of excitons on the highlying acceptor or donor state of energy $E_{i 1}=5.7 \mathrm{eV} .{ }^{17}$ In the framework of this hypothesis the dissociation of lowerenergy excitons is energetically forbidden. Indeed, the energy gain from the charge localization on donor or acceptor is $E_{\mathrm{ex}}-E_{i 1}$, where $E_{\mathrm{ex}} \equiv E_{i 2}$ is the exciton energy. For the efficient charges separation, this gain in energy has to be higher than the exciton binding energy $E_{g}-E_{\mathrm{ex}}$. This allows the energy balance equation relative to the process threshold,

$$
E_{g}-E_{\mathrm{ex}}=E_{\mathrm{ex}}-E_{i 1},
$$




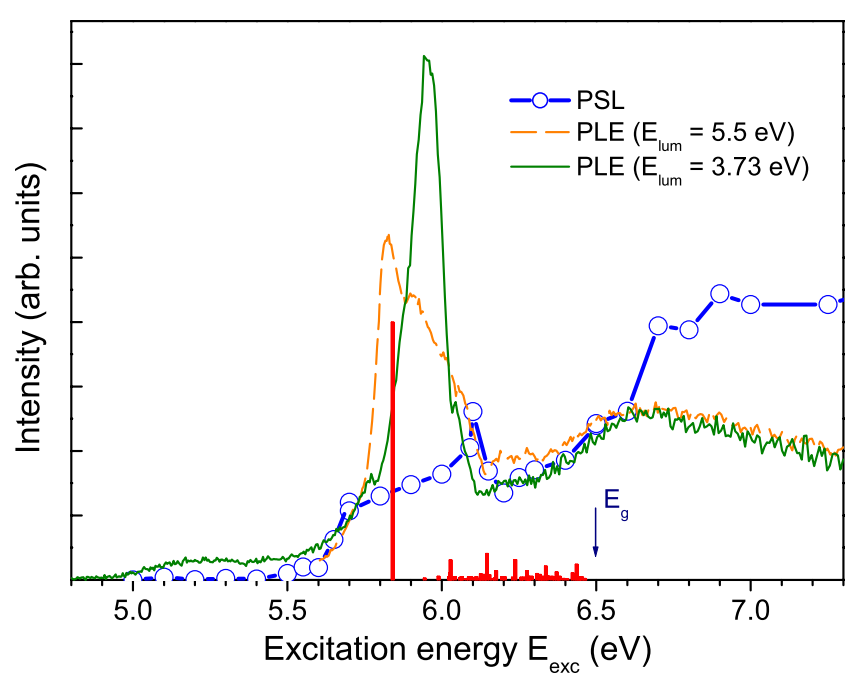

FIG. 7. (Color online) PSL excitation spectrum (open circles) and PLE spectra of bound exciton luminescence (5.5 eV, dashed line) and deep DAP luminescence (3.73 eV, solid line). Theoretical oscillator strengths (Refs. 34, 60, and 61) of free exciton transitions in $\mathrm{hBN}$ are shown by straight vertical bars.

$$
E_{g}=2 E_{i 2}-E_{i 1}=6.5 \mathrm{eV} .
$$

Interestingly, both alternatives in identification of the spectral feature at $E_{i 2}=6.1 \mathrm{eV}$ result in the same band-gap energy of $E_{g}=6.5 \mathrm{eV}$.

Our estimation of the hBN band-gap energy is in good agreement with that obtained from all-electron $G W$ calculations recently performed by Arnaud et al. ${ }^{34}$ Moreover, taking the exciton transitions at $5.8 \mathrm{eV}$, we obtain the exciton binding energy of $0.7 \mathrm{eV}$. This disagrees with the Wannier-type exciton suggested by Watanabe et al. ${ }^{1}$ and supports Arnaud's prediction of the Frenkel-type exciton with strong binding energy. ${ }^{34}$

The shape analysis of PLE and PSL excitation spectra supports the obtained value of hBN band-gap energy. In Fig. 7 we have plotted the PLE spectra of the bound excitons luminescence $\left(E_{\text {lum }}=5.5 \mathrm{eV}\right)$ together with the PLE spectra of broad DAP luminescence $\left(E_{\text {lum }}=3.73 \mathrm{eV}\right)$. The PLE $\left(E_{\text {lum }}=5.5 \mathrm{eV}\right)$ peak at $5.8 \mathrm{eV}$ fits well the position of the strongest excitonic transitions predicted theoretically, $34,60,61$ as represented by vertical bar in Fig. 7. As well the plateau of the PLE spectra in the energy range between 6.1 and $6.5 \mathrm{eV}$ is related to low-intensity excitonic transitions converging to the dissociation limit. Interestingly, both PLE spectra coincide in the high-energy spectra range above $6.1 \mathrm{eV}$ and follow the PSL spectrum until $6.5 \mathrm{eV}$. Above $6.5 \mathrm{eV}$ the PSL grows faster while the intensity of the PLE spectra decreases.
The PSL is expected to increase at $h \nu>E_{g}$ where, according to Fig. 4, process I efficiently contributes to the distant traps population. This difference in shapes between the PLE and PSL spectra is therefore explained by interband transitions and supports well our band-gap energy estimation $E_{g}$ $=6.5 \mathrm{eV}$.

Our final remark concerns the shape of PLE spectrum. From a general point of view, as long as the hBN can be considered as optically thin material, the shape of the PLE spectrum is expected to follow the intrinsic absorption spectrum. At higher absorbance, a saturation or even a decrease in PLE intensity appears as an intensification of nonradiative recombination processes. Interestingly, we observe the indication of saturation effect around $6.5 \mathrm{eV}$ in good agreement with our band-gap estimation.

\section{CONCLUSION}

Photoluminescence of hexagonal boron nitride has been studied by means of the time- and energy-resolved photoluminescence spectroscopy methods. Depending on the excitation energy several luminescence bands have been observed. (i) A strongly structured band in the energy range between 4.1 and $3.3 \mathrm{eV}$ is assigned to phonon replicas of an impurity luminescence. (ii) A very broad band $(\Delta E \sim 1 \mathrm{eV})$ centered at $3.9 \mathrm{eV}$ is assigned to DAP transitions involving a strongly localized acceptor complex center. Moreover, the intensity ratio between these two emissions strongly depends on excitation photon energy. (iii) $q$-DAPs are responsible for the $5.3-\mathrm{eV}$ emission and (iv) emission of excitons bound to defects is observed at $5.5 \mathrm{eV}$.

The excitation of samples by high-energy photons above $5.4 \mathrm{eV}$ enables another phenomenon called photostimulated luminescence, which is due to distantly trapped photoinduced charges. PSL is observed in hBN following interband and acceptor- and donor-band transitions. Moreover, we show that in contrast to DAP, PSL can also result from a direct $q$-DAP excitation. The comparison of photoluminescence excitation and PSL spectra allows band-gap energy estimation of $6.5 \mathrm{eV}$ and supports the hypothesis of Frenkeltype exciton in hBN with large binding energy of $0.7 \mathrm{eV}$. These conclusions support most recent theoretical calculations. ${ }^{34,35}$

\section{ACKNOWLEDGMENTS}

This work was supported by the IHP Contract No. HPRICT-1999-00040 of the European Commission. The authors are grateful to G. Stryganyuk for assistance in conducting experiments at HASYLAB (synchrotron DESY) and to P. Jaffrennou and F. Ducastelle for helpful discussions.
${ }^{1}$ K. Watanabe, T. Taniguchi, and H. Kanda, Nat. Mater. 3, 404 (2004).

${ }^{2}$ Y. Kubota, K. Watanabe, O. Tsuda, and T. Taniguchi, Science 317, 932 (2007).
${ }^{3}$ R. S. Lee, J. Gavillet, M. Lamy de la Chapelle, A. Loiseau, J.-L. Cochon, D. Pigache, J. Thibault, and F. Willaime, Phys. Rev. B 64, 121405(R) (2001).

${ }^{4}$ R. Arenal, O. Stephan, J. L. Cochon, and A. Loiseau, J. Am. 
Chem. Soc. 129, 16183 (2007).

${ }^{5}$ A. Loiseau, F. Willaime, N. Demoncy, G. Hug, and H. Pascard, Phys. Rev. Lett. 76, 4737 (1996).

${ }^{6}$ N. G. Chopra, R. J. Luyken, K. Cherrey, V. H. Crespi, M. L. Cohen, S. G. Louie, and A. Zettl, Science 269, 966 (1995).

${ }^{7}$ P. Jaffrennou, F. Donatini, J. Barjon, J. S. Lauret, A. Maguer, B. Attal-Tretout, F. Ducastelle, and A. Loiseau, Chem. Phys. Lett. 442, 372 (2007).

${ }^{8}$ P. Jaffrennou, J. Barjon, J. S. Lauret, A. Maguer, D. Golberg, B. Attal-Trétout, F. Ducastelle, and A. Loiseau, Phys. Status Solidi B 244, 4147 (2007).

${ }^{9}$ P. Jaffrennou, J. Barjon, T. Schmid, L. Museur, A. Kanaev, J.-S. Lauret, C. Y. Zhi, C. Tang, Y. Bando, D. Golberg, B. AttalTretout, F. Ducastelle, and A. Loiseau, Phys. Rev. B 77, 235422 (2008).

${ }^{10}$ M. G. Silly, P. Jaffrennou, J. Barjon, J.-S. Lauret, F. Ducastelle, A. Loiseau, E. Obraztsova, B. Attal-Tretout, and E. Rosencher, Phys. Rev. B 75, 085205 (2007).

${ }^{11}$ K. Watanabe, T. Taniguchi, T. Kuroda, and H. Kanda, Appl. Phys. Lett. 89, 141902 (2006).

${ }^{12}$ K. Watanabe, T. Taniguchi, and H. Kanda, Phys. Status Solidi A 201, 2561 (2004).

${ }^{13}$ C. A. Taylor II, S. W. Brown, V. Subramaniam, S. Kidner, S. C. Rand, and R. Clarke, Appl. Phys. Lett. 65, 1251 (1994).

${ }^{14}$ V. L. Solozhenko, A. G. Lazarenko, J. P. Petitet, and A. V. Kanaev, J. Phys. Chem. Solids 62, 1331 (2001)

${ }^{15}$ D. A. Evans, A. G. McGlynn, B. M. Towlson, M. Gunn, D. Jones, T. E. Jenkins, R. Winter, and N. R. J. Poolton, J. Phys.: Condens. Matter 20, 075233 (2008).

${ }^{16}$ P. Jaffrennou, J. Barjon, J.-S. Lauret, B. Attal-Trétout, F. Ducastelle, and A. Loiseau, J. Appl. Phys. 102, 116102 (2007).

${ }^{17}$ L. Museur and A. V. Kanaev, J. Appl. Phys. 103, 103520 (2008).

${ }^{18}$ D. M. Hoffman, G. L. Doll, and P. C. Eklund, Phys. Rev. B 30, 6051 (1984).

${ }^{19}$ R. Geick, C. H. Perry, and G. Rupprecht, Phys. Rev. 146, 543 (1966).

${ }^{20}$ S. N. Grinyaev, F. V. Konusov, V. V. Lopatin, and L. N. Shiyan, Phys. Solid State 46, 435 (2004).

${ }^{21}$ G. Chen, X. Zhang, B. Wang, X. Song, B. Cui, and H. Yan, Appl. Phys. Lett. 75, 10 (1999).

${ }^{22}$ Z. Remes, M. Nesladek, K. Haenen, K. Watanabe, and T. Taniguchi, Phys. Status Solidi A 202, 2229 (2005).

${ }^{23}$ A. Mansour and S. E. Schnatterly, Phys. Rev. B 36, 9234 (1987).

${ }^{24}$ R. D. Carson and S. E. Schnatterly, Phys. Rev. Lett. 59, 319 (1987).

${ }^{25}$ J. B. MacNaughton, A. Moewes, R. G. Wilks, X. T. Zhou, T. K. Sham, T. Taniguchi, K. Watanabe, C. Y. Chan, W. J. Zhang, I. Bello, S. T. Lee, and H. Hofsäss, Phys. Rev. B 72, 195113 (2005).

${ }^{26}$ J. Serrano, A. Bosak, R. Arenal, M. Krisch, K. Watanabe, T. Taniguchi, H. Kanda, A. Rubio, and L. Wirtz, Phys. Rev. Lett. 98, 095503 (2007).

${ }^{27}$ J. A. Carlisle, E. L. Shirley, L. J. Terminello, J. J. Jia, T. A. Callcott, D. L. Ederer, R. C. C. Perera, and F. J. Himpsel, Phys. Rev. B 59, 7433 (1999)

${ }^{28}$ J. J. Jia, T. A. Callcott, E. L. Shirley, J. A. Carlisle, L. J. Terminello, A. Asfaw, D. L. Ederer, F. J. Himpsel, and R. C. C. Perera, Phys. Rev. Lett. 76, 4054 (1996).

${ }^{29}$ B. M. Davies, F. Bassani, F. C. Brown, and C. G. Olson, Phys.
Rev. B 24, 3537 (1981).

${ }^{30}$ F. C. Brown, R. Z. Bachrach, and M. Skibowski, Phys. Rev. B 13, 2633 (1976)

${ }^{31}$ C. Tarrio and S. E. Schnatterly, Phys. Rev. B 40, 7852 (1989).

${ }^{32}$ R. D. Leapman, P. L. Fejes, and J. Silcox, Phys. Rev. B 28, 2361 (1983).

${ }^{33}$ R. D. Leapman and J. Silcox, Phys. Rev. Lett. 42, 1361 (1979).

${ }^{34}$ B. Arnaud, S. Lebègue, P. Rabiller, and M. Alouani, Phys. Rev. Lett. 96, 026402 (2006).

${ }^{35}$ L. Wirtz, A. Marini, M. Gruning, and A. Rubio, arXiv:cond-mat/ 0508421 (unpublished).

${ }^{36}$ G. Zimmerer, Nucl. Instrum. Methods Phys. Res. A 308, 178 (1991).

${ }^{37}$ V. Nagirnyi, M. Kirm, A. Kotlov, A. Lushchik, and L. Jönsson, J. Lumin. 102-103, 597 (2003).

${ }^{38}$ V. Nagirnyi, P. Dorenbos, E. Feldbach, L. Jönsson, M. Kerikmäe, M. Kirm, E. v. d. Kolk, A. Kotlov, H. Kraus, A. Lushchik, V. Mikhailik, R. Sarakvasha, and A. Watterich, in Proceedings of the Eighth International Conference on Inorganic Scintillators and their use in Scientific and Industrial Applications SCINT 05 (Alushta, Crimea, Ukraine, 2005).

${ }^{39}$ L. Museur, D. Anglos, J. P. Petitet, J. P. Michel, and A. V. Kanaev, J. Lumin. 127, 595 (2007).

${ }^{40}$ B. Berzina, L. Trinckler, R. Krutohvostov, R. T. Williams, D. L. Caroll, R. Czerw, and E. Shishnonok, Phys. Status Solidi C 2, 318 (2005)

${ }^{41}$ A. V. Kanaev, J. P. Petitet, L. Museur, V. Marine, V. L. Solozhenko, and V. Zafiropulos, J. Appl. Phys. 96, 4483 (2004).

${ }^{42}$ J. Wu, W.-Q. Han, W. Walukiewicz, J. W. Ager III, W. Shan, E. E. Haller, and A. Zettl, Nano Lett. 4, 647 (2004).

${ }^{43}$ A. I. Lukomskii, V. B. Shipilo, and L. M. Gameza, J. Appl. Spectrosc. 57, 607 (1992).

${ }^{44}$ M. Kawaguchi, Y. Kita, and M. Doi, J. Mater. Sci. 26, 3926 (1991).

${ }^{45}$ K. Era, F. Minami, and T. Kuzuba, J. Lumin. 24-25, 71 (1981).

${ }^{46}$ S. Larach and R. E. Shrader, Phys. Rev. 102, 582 (1956).

${ }^{47}$ S. Larach and R. E. Shrader, Phys. Rev. 104, 68 (1956).

${ }^{48}$ L. Museur, J. P. Petitet, J. P. Michel, W. Marine, D. Anglos, C. Fotakis, and A. Kanaev, J. Appl. Phys. (to be published).

${ }^{49}$ A. Katzir, J. T. Suss, A. Zunger, and A. Halperin, Phys. Rev. B 11, 2370 (1975).

${ }^{50}$ A. Zunger and A. Katzir, Phys. Rev. B 11, 2378 (1975).

${ }^{51}$ T. Taniguchi and K. Watanabe, J. Cryst. Growth 303, 525 (2007).

${ }^{52}$ G. Kern, G. Kresse, and J. Hafner, Phys. Rev. B 59, 8551 (1999).

${ }^{53}$ L. Museur and A. Kanaev (unpublished).

${ }^{54}$ L. Pavesi and M. Guzzi, J. Appl. Phys. 75, 4779 (1994).

${ }^{55}$ H. Samelson and A. Lempicki, Phys. Rev. 125, 901 (1962).

${ }^{56}$ S. Shionoya, T. Koda, K. Era, and H. Fujiwara, J. Phys. Soc. Jpn. 19, 1157 (1964).

${ }^{57}$ E. W. Williams, Phys. Rev. 168, 922 (1968).

${ }^{58}$ M. A. Reshchikov and H. Morkoc, J. Appl. Phys. 97, 061301 (2005).

${ }^{59}$ J. W. Allen, Semicond. Sci. Technol. 10, 1049 (1995).

${ }^{60}$ L. Wirtz, A. Marini, M. Grüning, C. Attaccalite, G. Kresse, and A. Rubio, Phys. Rev. Lett. 100, 189701 (2008).

${ }^{61}$ B. Arnaud, S. Lebègue, P. Rabiller, and M. Alouani, Phys. Rev. Lett. 100, 189702 (2008). 